By using a symmetrical arrangement of electrodes (Fig. 1c) it was also possible to make good silver-silver joints by partially melting two wires together. These joints again were mechanically strong but there was found to be a reduction of the rrr of the silver at the joint. As this reduction was only $\sim 30 \%$ and was confined to $\sim 1 \mathrm{~cm}$ on either side of the joint, the effect was not serious.

\section{Reference}

1 Bradley, D.I., Guénault, A.M., Keith, V., Pickett, G.R., Pratt, W.P. Jr. J Phys C Lett (1982)

\title{
A microprocessor based, multi-channel low-temperature monitoring system
}

\author{
B.W. Kuiper and M.H.H. van Dijk
}

Key words: microprocessors, thermo-resistive sensors, monitoring system

Modern microelectronics has found its way into cryogenic temperature measurements and control. Various systems are commercially available. ${ }^{1}$ In our group we have investigated the possibility of linking thermo-resistive sensors to an existing modular microprocessor system. This has been done in order to develop an automatic multichannel monitoring system.

The basic idea is that of a standard data acquisition system (Fig. 1). Under microprocessor control, successive analogue signals are selected, conditioned and converted into digital values. These values are then stored, processed and sent to the desired output device. Cryogenic sensors give rise to special design requirements.

Self-heating should be low. A study of the measuring currents as recommended by the manufacturer of our germanium and carbon-glass sensors (Lake Shore Inc. ${ }^{2}$ ), makes it clear that the system should preferably be designed to operate with a constant excitation voltage rather than with a constant current. According to these data, an applied voltage of $10 \mathrm{mV}$ is the maximum permitted for all sensors, independent of the actual resistance value.

In our case an accuracy of $0.5 \%$ in the resistance measurement is required and this means that special attention has to be paid to the multiplexing, conditioning and analogue to digital conversion of the low level electric signals. The possible influence of leakage currents, contact potentials, thermal potentials and switch resistances has to be recognized. Switching elements may be low-leakage CMOS

The authors are working in the Low Temperature Group of the Physics Department of the Twente University of Technology, PO Box 217, 7500 AE Enschede, The Netherlands. Paper received 10 June 1982 .

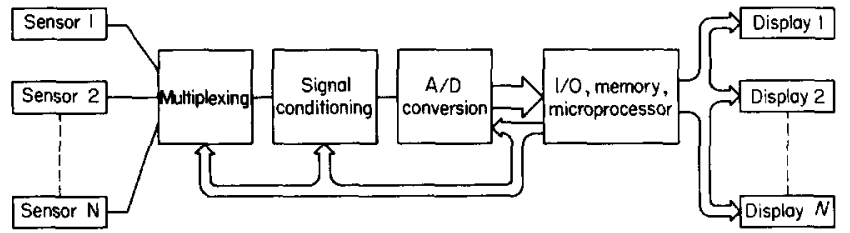

Fig. 1 Basic idea of the monitoring system

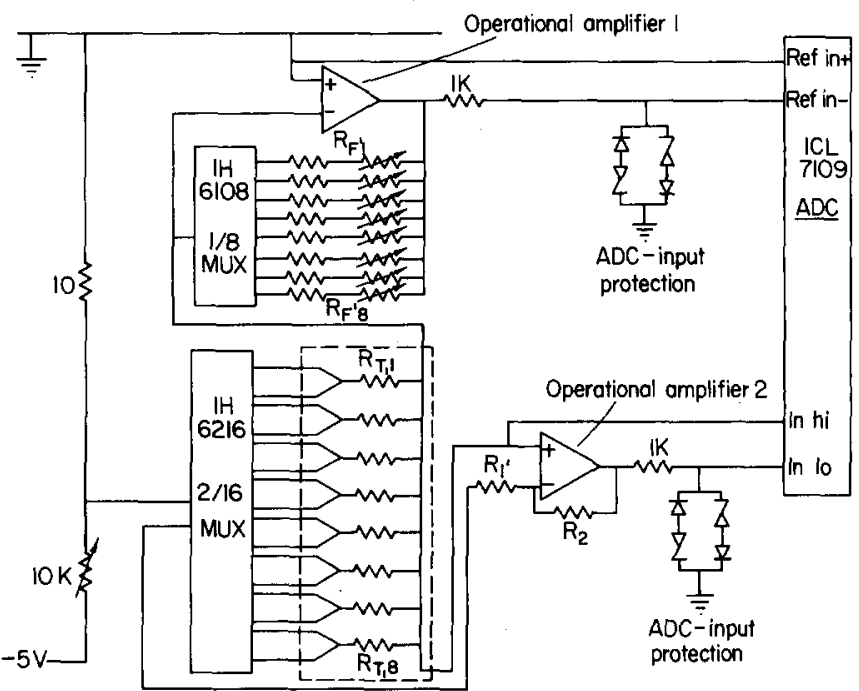

Fig. 2 The multiple-sensor interface. The part within the dotted line is inside the cryostat

analogue multiplexers or so-called 'low thermal EMF' reed relais.

We shall first confine ourselves to the eight-channel sensor interface that we designed. After that calibration polynomials, the microprocessor system and microprocessor programme will be dealt with briefly.

\section{The multiple-sensor interface}

The circuit of this interface is given schematically in Fig. 2 . The analogue multiplexers are the Intersil IH 6108 (8 channel) and IH 6216 (8 channel differential). These IC's have been chosen because of their low leakage current.

The analogue to digital converter (ADC) is the Intersil ICL 7109 , a 12 bits dual slope integrating $\mathrm{ADC}$. The operational amplifiers are the OPOS of PMI.

Each channel can be selected by offering the appropriate digital information to the three select inputs and the chip- 


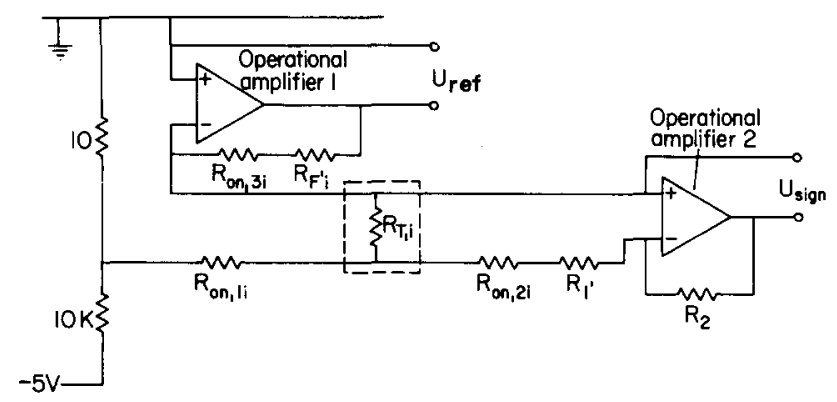

Fig. 3 One-channel circuit of the multiplexed system

enables of the analogue multiplexers. The effective circuitry, with one of the channels selected is shown in Fig. 3.

$R_{\mathrm{Ti}}$ is the sensor selected. $R_{\text {on, } 1 \mathrm{i}}$ and $R_{\text {on, } 2 \mathrm{i}}$ are the onresistances of the IH 6216 and $R_{\text {on }, 3 \mathrm{i}}$ is the on-resistance of the IH 6108. $R_{F_{i}^{\prime}}$ and $R_{1}^{\prime}$ both consist of a fixed resistor and a precision potentiometer in series.

For convenience we write:

$$
\begin{aligned}
& R_{\text {on }, 2 \mathrm{i}}+R_{1}^{\prime}=R_{1} \\
& R_{\text {on }, 3 \mathrm{i}}+R_{\mathrm{F}^{\prime} \mathrm{i}}=R_{\mathrm{Fi}}
\end{aligned}
$$

Let the voltage across the sensor be $U_{0}$, then the following relations yield:

$$
\begin{aligned}
& U_{0}=i \cdot R_{\mathrm{Ti}} \\
& U_{\text {ref }}=i \cdot R_{\mathrm{Fi}} \\
& U_{\text {sign }}=U_{0} \cdot \frac{R_{2}}{R_{1}}
\end{aligned}
$$

The value of $R_{\mathrm{Ti}}$ can be resolved by making use of the ratiometric property of the ADC.

After connecting $U_{\text {ref }}$ and $U_{\text {sign }}$ to the differential reference and signal input of this $\mathrm{ADC}$, the following relation yields for the digital output: ${ }^{3}$

$$
C_{\mathrm{n}}=2048 \cdot \frac{U_{\text {sign }}}{U_{\text {ref }}}
$$

with (1) and (2):

$$
C_{\mathrm{n}}=2048 \cdot \frac{1}{R_{\mathrm{Fi}}} \cdot \frac{R_{2}}{R_{1}} \cdot R_{\mathrm{Ti}}
$$

This means that $R_{\mathrm{Ti}}$ can be found from the ADC output as follows:

$$
R_{\mathrm{Ti}}=S_{\mathrm{i}} \cdot C_{\mathrm{n}}{ }^{\prime}
$$

with $S_{\mathrm{i}}$ depending of $R_{1}, R_{2}$ and $R_{\mathrm{Fi}}$ :

$$
S_{\mathrm{i}}=\frac{R_{\mathrm{Fi}}}{2048} \cdot \frac{R_{1}}{R_{2}}
$$

The way to determine $S_{\mathrm{i}}$ and $R_{\mathrm{Fi}}$ for a particular sensor, has been worked out in the Appendix.

An integrating type ADC suppresses noise and disturbances to a large extent. ${ }^{3}$ As $R_{\mathrm{Fi}}$ can be adjusted for each channel to give the proper ADC-output, any systematic error term that gives a linear contribution to this output can be eliminated. Error terms that contribute systematically to the offset of the ADC-output, can be minimized by adjusting the offset of operational amplifier 2 .

An accuracy of better than $0.5 \%$ in the $R$-measurements was obtained over the major domain of ADC outputs.
Once present in the microprocessor system, the digital values of the sensor resistances have to be converted in temperature values.

\section{Temperature-resistance}

In our test system we restricted ourselves to germanium and carbon-glass resistors (types CGR-1 -500, GR-500, GR-200A-500 and GR-2000A-1000). We found the following polynomials GR $200 \mathrm{H}-1000$ fit the calibration results very satisfactorily:

$$
\begin{aligned}
& \text { Germanium: } \ln T=A+B \ln \mathrm{R}+C(\ln R)^{2}+D(\ln R)^{3} \\
& \text { Carbon-glass: } T^{-1}=A+B \ln R+C(\ln R)^{2}+D(\ln R)^{3}
\end{aligned}
$$

An equation similar to 5 is recommanded by Leung and $\mathrm{Kos}^{4}$ for not too large temperature intervals.

Relation 6 is in agreement with the one proposed by Zimmerman and Hoare 5 for carbon-glass resistors. The coefficients $A_{\mathrm{i}}, B_{\mathrm{i}}, C_{\mathrm{i}}$ and $D_{\mathrm{i}}$ of the polynomials were determined using a least-square curve-fitting program. These coefficients have to be present in the microprocessor memory, in addition to values of $S_{\mathrm{i}}(4)$ and information about the type of sensor, GR or CGR.

\section{The microprocessor system}

The minimum system that serves as a stand-alone eightchannel monitoring system, consists of the following components: the multiple-sensor interface already described; a central processing unit (CPU), the Intel 8085 microprocessor, with a circuit to drive the microprocessor bus; 4 Kbyte of programmable read-only memory (PROM), for program and fixed-data storage, $0.5 \mathrm{Kbyte}$ of randomaccess memory (RAM), for the storage of temporary data; 22 general purpose input/output (I/O) lines for communication with the multiple-sensor interface; 2 byte of dipswitch memory, giving the user the possibility of choosing one of several modes of operation. A multiple display interface, which is able to drive eight four-digit seven-segment displays. This interface has been designed around two Intel 8279 display interface IC's.

\section{Program structure}

The structure of the microprocessor program is shown in Fig. 4. In the measuring routine an input channel is ad-

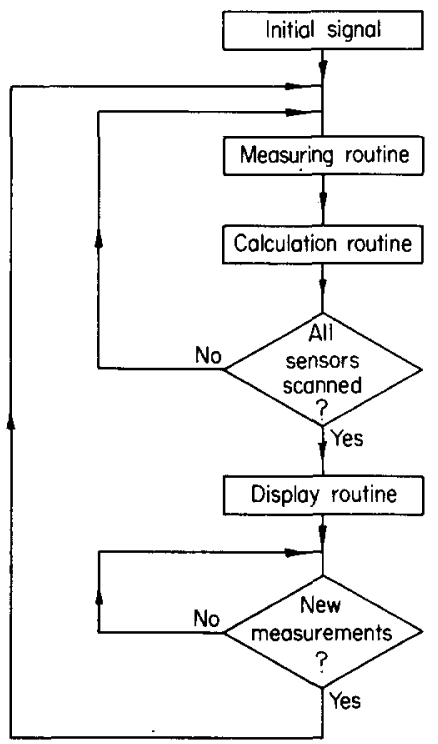

Fig. 4 Schematic struct ure of the microprocessor program 
dressed, an analogue to digital conversion is performed and the results are stored in memory locations.

The calculation routine utilizes a set of hexa-decimal floating-point routines to evaluate (3), (5) and (6). The resulting $R$ and $T$ values are stored in floating-point BCD format. In the meantime the conversion on the next channel has already been started. Eight switch settings of dipswitch memory give the user the choice of either $R$ or $T$ display for each channel. In the display routine the desired values are converted into seven-segment codes and sent to the display interfaces.

\section{Discussion and conclusion}

During testing, the sensors were in vacuum (about $10^{-2} \mathrm{~Pa}$ ) and attached to parts of an experimental maser configuration.

The measuring voltage could be increased to $20 \mathrm{mV}$, without significant sensor self-heating effects, see Fig. 5. Only spurious effects of thermal potentials were observed.

Oscillations of the polynomials outside the calibration interval may give rise to misleading $T$ readings. It is therefore recommended that the temperature traject up to $300 \mathrm{~K}$ be globally covered by the $R / T$ conversion of the system. A seperate second-degree polynomial of the same type (5) or (6) can be helpful to deal with the tale of the $R / T$ curve, see Fig. 6 . This polynomial should be used for $C_{\mathrm{n}}<C_{\max } / D$ (see appendix).

The system presented offers convenient temperature monitoring in cryogenic experiments. Interfacing with control units, alarm systems or penwriters is easy to accomplish.

The authors wish to express their appreciation to Mr. B. van den Dolder of the Electronics Department for his advice conceming the microprocessor system. They would also like to thank the Messrs. Enzing, Van der Vecht, Van Lieburg, Kamp, Oude Alink and Scholte Lubberink for their help and careful measurements.

\section{Appendix}

Determination of the $S_{\mathrm{i}}$ and $R_{\mathrm{Fi}}$ of a sensor. Let Fig. 6 represent the $R-T$ behaviour of sensor $i$. A maximum and a minimum useful value are assigned to it: $R_{\mathrm{Ti}, \max }$ and

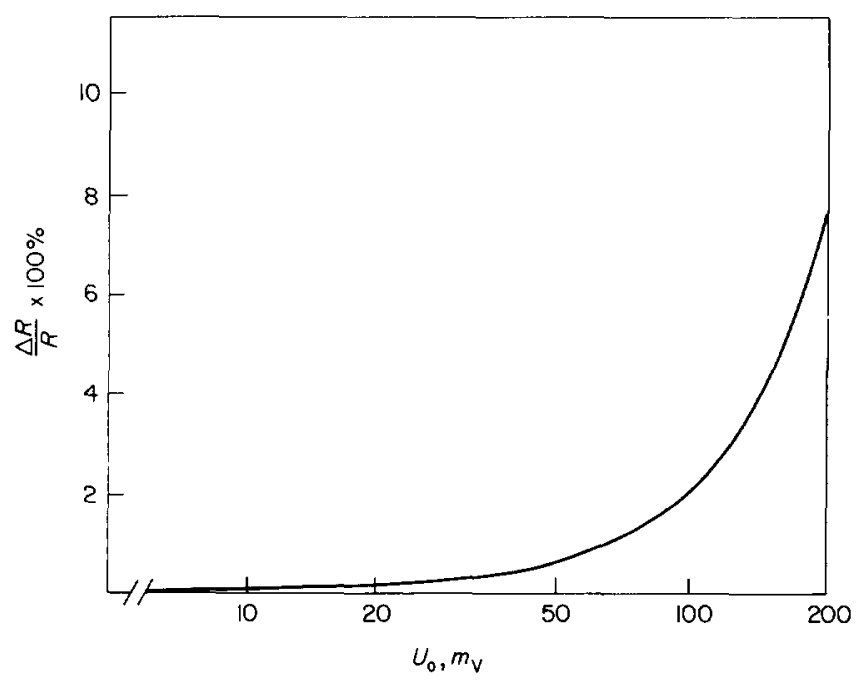

Fig. 5 The error in the detected resistance value - due to sensor self heating - as a function of the excitation voltage

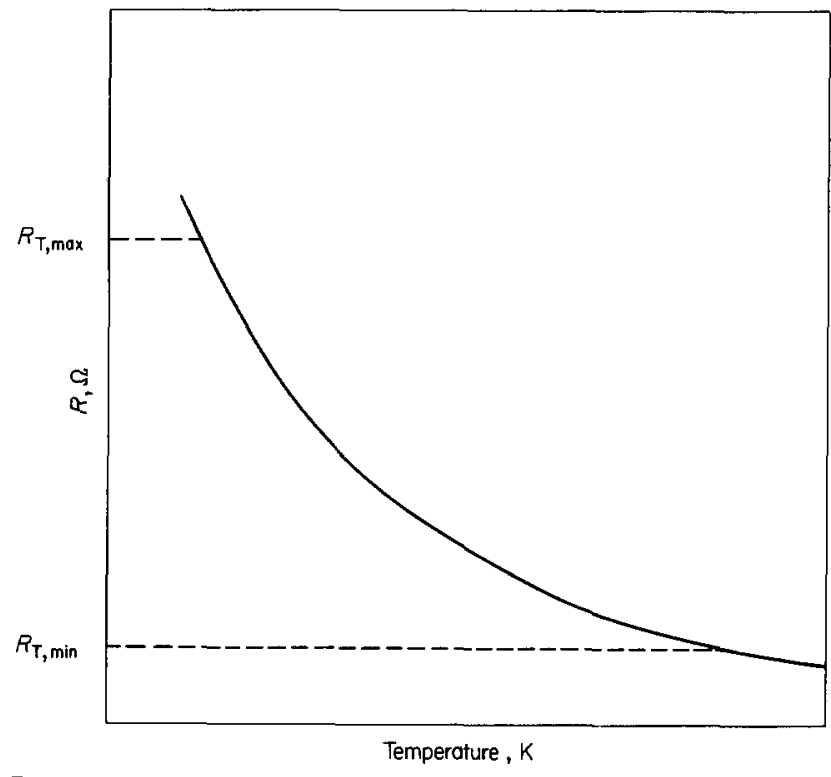

Fig. 6 The $R-T$ behaviour of a sensor

$R_{\mathrm{Ti} \text { min }}$ respectively. The value of $S_{\mathrm{j}}$, for optimal use of the ADC output range, can be found from (3), with $C_{\mathrm{n}, \max }=4096$ :

$$
S_{\mathrm{i}}=\frac{R_{\mathrm{Ti}, \max }}{4096}
$$

$R_{\mathrm{Fi}}$ can be found from (4), after a value for $R_{2} / R_{1}$ has been chosen, This $R_{2} / R_{1}$ value is limited by the maximum values that $U_{\text {sign }}$ and $U_{\text {ref }}$ may take. Let the maximum value for both these ADC-inputs be $U_{\mathrm{M}}$ (somewhat smaller than the positive supply voltage of the ADC).

(2) gives: $\frac{R_{2}}{R_{1}} \leqslant \frac{U_{\mathrm{M}}}{U_{0}}$

and $i_{\max }=U_{0} / R_{\mathrm{T}, \min }$ with (1), (4) and (7) gives:

$$
\frac{R_{2}}{R_{1}} \leqslant 2, \frac{U_{\mathrm{M}}}{U_{0} \cdot D},
$$

where $D$ is defined as:

$$
D=\frac{R_{\mathrm{Ti}, \max }}{R_{\mathrm{Ti}, \min }} .
$$

In our test system we took: $U_{\mathrm{M}}=4 \mathrm{~V}, U_{0}=10 \mathrm{mV}, D=10$. In this case we find:

$$
\frac{R_{2}}{R_{1}} \leqslant 80 \text {. }
$$

$R_{\text {Fi }}$ can now be calculated, using (4) and (7):

$$
R_{\mathrm{Fi}}=\frac{R_{\mathrm{Ti}, \max }}{2} \cdot \frac{R_{2}}{R_{1}}
$$

\section{References}

1 Lake Shore Cryotronics Inc. USA, Models CST-900, DRC-800 Thor Cryogenics, England, Models E3010, E3050, etc. Scientific Instruments, Lake Worth, Florida USA), Series 1800. RV-Elektronika OY (Helsinki, Finland), System AVS-45

2 Lake Shore Cryotronics Inc., Series GR200A and CGR-1 data sheets

3 Intersil, Data Acquisition Handbook (1980), 40, 44 and ICL 7109 data sheets

4 Leung, Y.K., Kos, J.F. Cryogenics 19 (1979) 531

5 Zimmerman, J.E., Hoare, F.E. Phys Chem Solids 17 (1960) 52 